\title{
Modelling and Control of Bounded Hybrid Systems in Power Electronics
}

\author{
Áron FEHÉR, Dénes Nimród KUTASI \\ Department of Electrical Engineering, \\ Faculty of Technical and Human Sciences, \\ Sapientia University, Tg. Mureș, \\ e-mail: fehera@ms.sapientia.ro,kutasi@ms.sapientia.ro
}

Manuscript received October 22, 2017; revised December 18, 2017.

\begin{abstract}
In this work, an explicit Model Predictive Control algorithm is devised and compared to classical control algorithms applied to a series resonant DC/DC converter circuit. In the first part, a model of the converter as a hybrid system is created and studied. In the second part, the predictive algorithm is applied and tested on the model. Finally, the designed control algorithm is compared to classical PI and sliding mode controllers.
\end{abstract}

Keywords: hybrid modelling, piecewise affine model, resonant converter, model predictive control

\section{Introduction}

These days nearly every time/response critical process is controlled with an embedded system. The high demands to achieve reliable performance in complex systems required the development of new control methods. To control a process, the properties of the said process must be known.

The term Hybrid Systems is relatively young in System theory. Systems which belong to this category cannot be categorised as fully continuous, nor fully discrete systems. From this definition we can conclude that hybrid systems are a mix of both, combining continuous and discrete events. Often these systems contain an analogue continuous-time process, some discrete stimuli, and a discrete controller.

A Hybrid system can be described as Piecewise Affine (PWA) system, which forms a particular class of nonlinear systems, where each state and output map are piecewise affine, or linear on each polyhedral partition of the state-input polytope [1]. Formula (1) shows the description of a PWA system, where $\underline{x}[k] \in$ 
$\mathbb{R}^{n}, \underline{y}[k] \in \mathbb{R}^{p}, \underline{u}[k] \in \mathbb{R}^{m}$ are the state, output and input vectors respectively, $A_{i}$, $B_{i}, C_{i}, D_{i}$, are the system matrices with the appropriate dimensions, $f_{i}, g_{i}$ are constant vectors and $P_{i}$ is the input-state polyhedron for $i$ discrete states.

$$
\left\{\begin{array}{c}
\underline{x}[k+1]=A_{i} \underline{x}[k]+B_{i} \underline{u}[k]+f_{i} \\
\underline{y}[k]=C_{i} \underline{x}[k]+D_{i} \underline{u}[k]+g_{i}
\end{array},(\underline{x}[k] \quad \underline{u}[k])^{T} \in P_{i}, i=\overline{1, k}\right.
$$

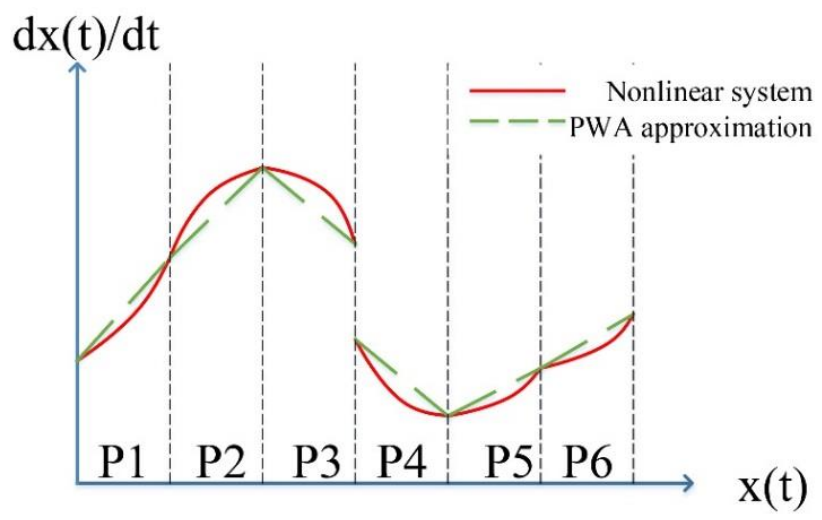

Figure 1: The figure shows the PWA approximation of a non-linear system containing discontinuities such as state changes or boundaries

Another description method is the Mixed Logic Dynamic (MLD) System, which is computer oriented and is widely used for controller synthesis. An MLD system can be written as shown in Equation (2), where $x[k] \in \mathbb{R}^{n} x\{0,1\}^{n}$, $y[k] \in \mathbb{R}^{p} \boldsymbol{x}\{0,1\}^{p}, \underline{u}[k] \in \mathbb{R}^{m} \boldsymbol{x}\{0,1\}^{m b}$ are the new concatenated state, input and output vectors, while $\underline{\delta} \in\{0,1\}^{r b}$, and $\underline{z} \in \mathbb{R}^{r r}$ are auxiliary variables.

$$
\left\{\begin{array}{l}
\underline{x}[k+1]=A_{1} \underline{x}[k]+B_{1} \underline{u}[k]+B_{2} \underline{\delta}[k]+B_{3} \underline{z}[k] \\
\underline{y}[k]=C_{1} \underline{x}[k]+D_{1} \underline{u}[k]+D_{2} \underline{\delta}[k]+D_{3} \underline{z}[k] \\
E_{2} \underline{\delta}[k]+E_{3} \underline{z}[k] \geq E_{1} \underline{x}[k]+E_{1} \underline{u}[k]+E_{1}
\end{array}\right.
$$

\section{Series Resonant Converter model}

In this section, the modelling of the Series Resonant DC/DC Converter (SRC) shown in Fig. 2 will be presented. 


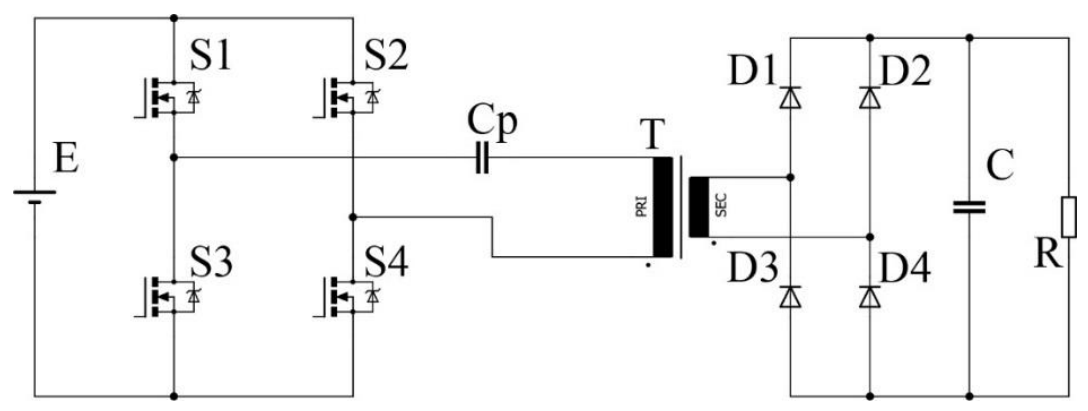

Figure 2: The figure shows an SRC circuit. The resonant tank and the output load acts as a voltage divider. The SRC can operate without load, but in that case, the output voltage can't be regulated

An equivalent circuit must be devised for the transformer, to create the converter model. The transformer equivalent circuit is deduced neglecting the magnetizing and core loss currents.

Many description methods were successfully used to model resonant converters, for example, discrete time model [2], continuous time model based on averaging methods [3], or with the progressive analysis of circuit waveforms [4]. The models are nonlinear. Hence it is common to use the small signal linearized approximation of the model around the operating point. The problem with linearized model is the invariance to perturbation, input fluctuation and load changes cannot be revealed. We have chosen the hybrid modelling as a description technique of the resonant converter.

The SRC can be represented as shown in Fig. 3 based on the equivalent circuit of the transformer. The $\mathrm{H}$ bridge switches are operated symmetrically (S1 and S4 are on, while S2 and S3 are off; S1 and S4 are off, while S2 and S3 are on).

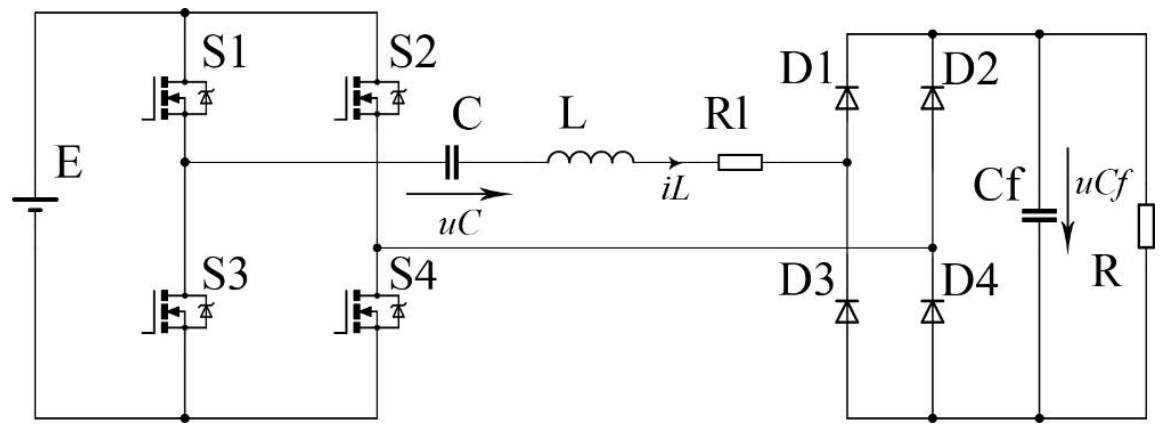

Figure 3: DC-DC SRC circuit with primary side approximate representation of the transformer 
Let the state space vector be $\underline{x}(t)=\left(\begin{array}{lll}i_{L} & u_{C} & u_{C f}\end{array}\right)^{T}$, the PWA representation is shown in Equation (3), with variables shown in Table 1. as discussed in [6].

$$
\left\{\begin{array}{c}
\dot{x}(t)=A_{i} \underline{x}(t)+f_{i} \\
i=R(u(t), \underline{x}(t))
\end{array}\right.
$$

The described system must be discretized to design an MPC algorithm. The sampling period must be chosen according to Shannon's sampling theorem. The resonant frequency of the resonant tank can be calculated as $f_{n}=\frac{1}{2 \pi \sqrt{L C}}$. In this study, the sampling period is chosen as $T_{s}=\frac{1}{10 f_{s}}$.

Let us consider the upper mentioned DC-DC SRC system with $\mathrm{L}=14.7 \mu \mathrm{H}$, $\mathrm{C}=560 \mathrm{nF}, \mathrm{R}_{\mathrm{L}}=0 \Omega, \mathrm{C}_{\mathrm{f}}=1 \mathrm{mF}, \mathrm{E}=48 \mathrm{~V}$ parameters. The resonant frequency of the system is $\mathrm{f}_{\mathrm{n}}=55.471 \mathrm{kHz}$, so that the sampling period will be $\mathrm{T}_{\mathrm{s}}=1.8 \mu \mathrm{s}$. Fig. 4 shows the open loop operation of the DC-DC converter created with Simscape Power Systems and Hysdel/MPT toolbox simulated with a $10 \mathrm{kHz}$ frequency and $50 \%$ duty cycle square wave input signal.

Table 1-PWA partition of the SRC based on the semiconductor states

\begin{tabular}{|c|cc|c|c|}
\hline$i$ & $A_{i}$ & $f_{i}$ & $R$ \\
\hline 1 & $\left(\begin{array}{ccc}-\frac{R_{L}}{L} & -\frac{1}{L} & -\frac{1}{L} \\
\frac{1}{C} & 0 & 0 \\
\frac{1}{C_{f}} & 0 & -\frac{1}{R C_{f}}\end{array}\right)$ & $\left(\begin{array}{ccc}\frac{E}{L} & 0 & 0\end{array}\right)^{T}$ & If $u(t)=1 \& x_{I}(t)>0$ \\
\hline 2 & $\left(\begin{array}{ccc}-\frac{R_{L}}{L} & -\frac{1}{L} & \frac{1}{L} \\
\frac{1}{C} & 0 & 0 \\
\frac{1}{C_{f}} & 0 & -\frac{1}{R C_{f}}\end{array}\right)$ & $\left(\begin{array}{ccc}\frac{E}{L} & 0 & 0\end{array}\right)^{T}$ & If $u(t)=1 \& x_{I}(t)<0$ \\
\hline
\end{tabular}




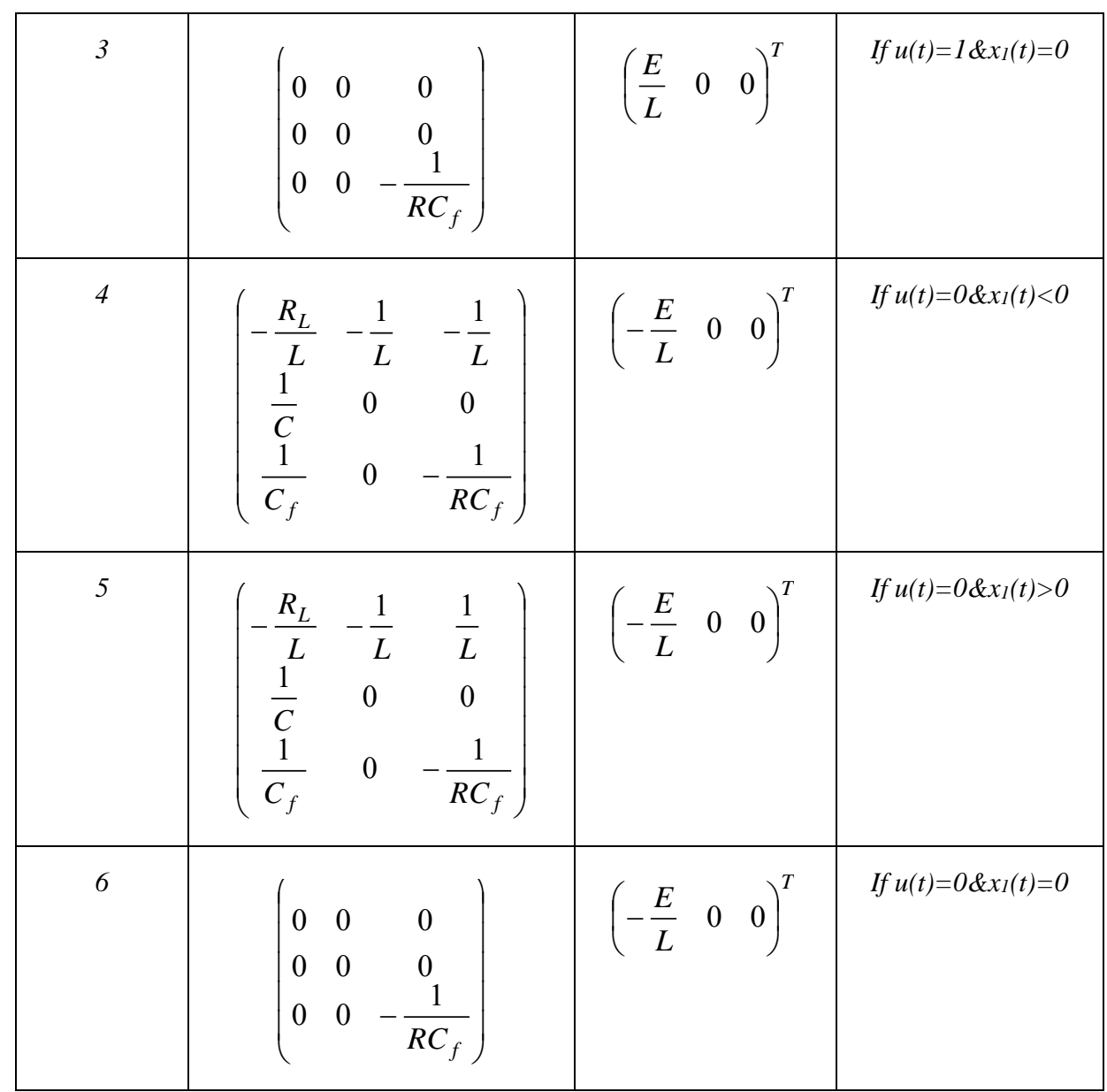

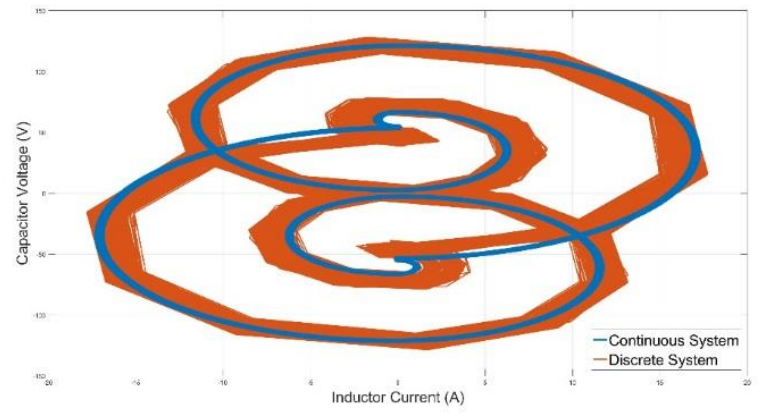

Figure 4: Comparison of the continuous SRC model created in Simscape Power Systems with the discretized version in Hysdel/MPT toolbox. The discrete model closely follows the state trajectory of the continuous model 


\section{Application of the explicit MPC}

The Model Predictive Control (MPC) is a particular case of constrained optimal control which predicts the optimal control signal for a given system for a given horizon. An infinite horizon sub-optimal controller can be designed by repeatedly solving finite time optimal control problems in receding horizon fashion. The resulting controller is referred to as Receding Horizon Controller (RHC). An RHC where the finite time optimal control law is computed by solving the optimization problem online is called MPC [5]. Solving online the Multi Parametric Quadratic Program (MPQP) at each time sample is a compute-intensive operation. The explicit version of the MPC developed in order to decrease the computation requirements.

The state boundaries of the hybrid SRC were deduced with a step function as an input signal. The HYSDEL PWA model was imported to MPT (Multi Parametric Toolbox) and was transformed to MLD model, with the boundaries attached to the states, and input as shown in Fig. 7. The online MPC was generated by solving the Multi Integer MPQP. For reference tracking, we used linear cost function, because the quadratic one failed to translate to S-function. The explicit version of the controller was generated with the help of the MPT toolbox, and the generated control algorithm was optimized by concatenating polyhedral partitions with the same control signal.

A series of tests were made with the designed controller. In Fig. 5 the closed loop system is tested with a constant load of $3 \Omega$, while the reference voltage is changed from $10 \mathrm{~V}$ to $12 \mathrm{~V}$ (The converter parameters are the same as in the last chapter). We can see, that steady state error is 0 in every case, but the overshoot is present. In Fig. 6 the reference voltage is kept at a constant $10 \mathrm{~V}$ while the load is changed. We can see, that the transient overshoot is present, but the controller tracks the reference with zero steady state error. Also, by increasing the prediction horizon, the overshoot decreases and the response time of the controller decreases. 


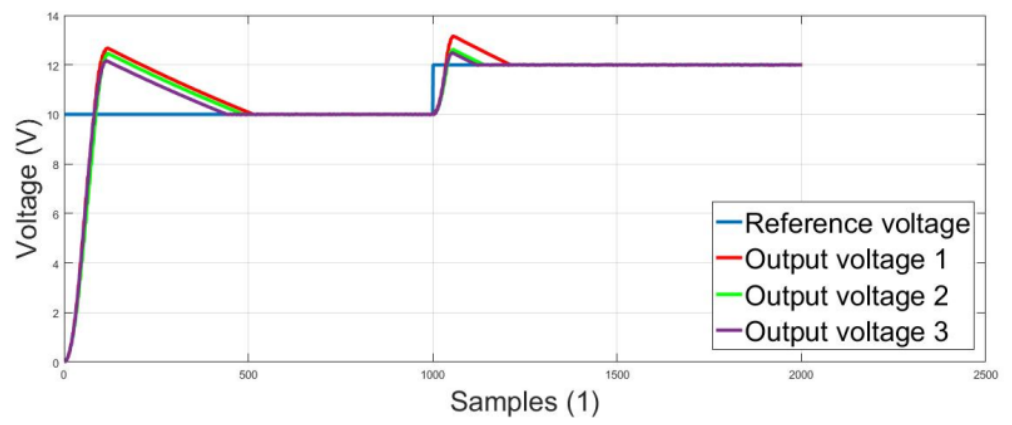

Figure 5: MPC applied to the SRC. In this scenario the reference voltage is perturbed, while the load is constant. The output voltages correspond to a controller with $\mathrm{N}=3,5$, and 10 prediction and control horizons respectively

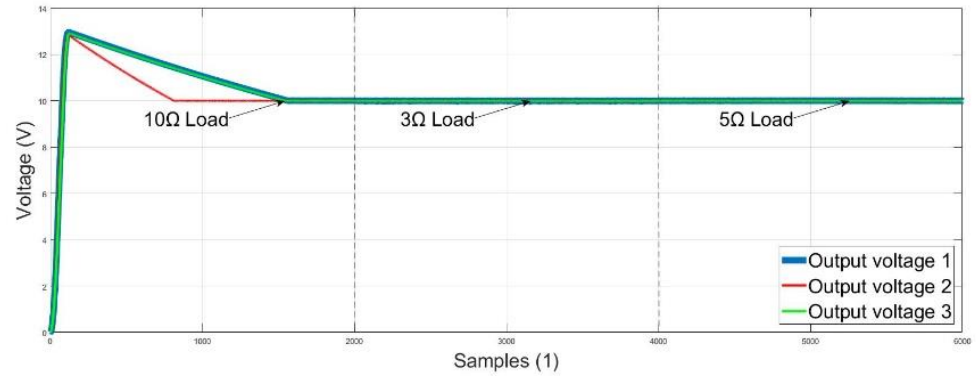

Figure 6: MPC applied to the SRC. In this scenario the reference voltage is constant, while the load is changed. The output voltages correspond to a controller with $\mathrm{N}=3,5$, and 10 prediction and control horizons respectively.

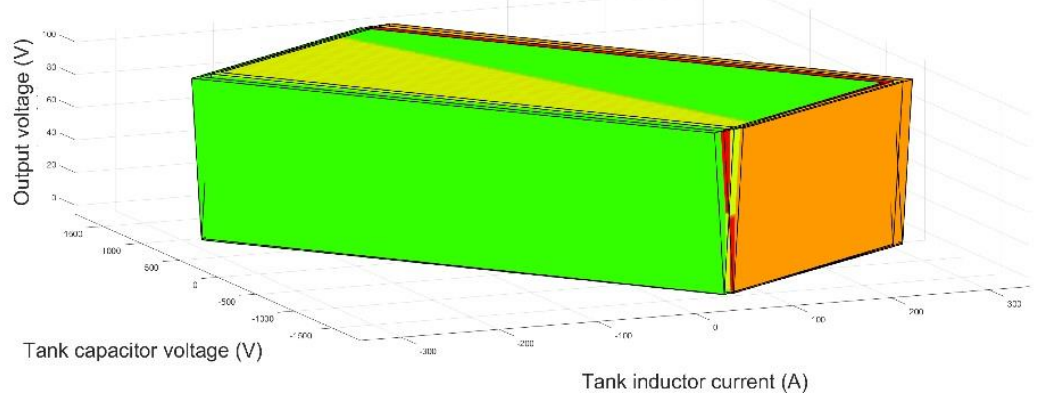

Figure 7: The figures show the reachable set of the SRC in the state space. With an input voltage of $48 \mathrm{~V}$, and a transformer ratio of $17: 1$ 


\section{Comparison of different control methods}

For comparison purposes, we designed two controllers for the converter. The first controller was a PI controller tuned to the linearized model of the SRC devised with the help of the Fourier series expansion of the nonlinear terms, keeping only the fundamental ones [7]. Fig. 8 shows the behaviour of the controlled system, from where we can conclude, the PI controller can be used for a given operating point.

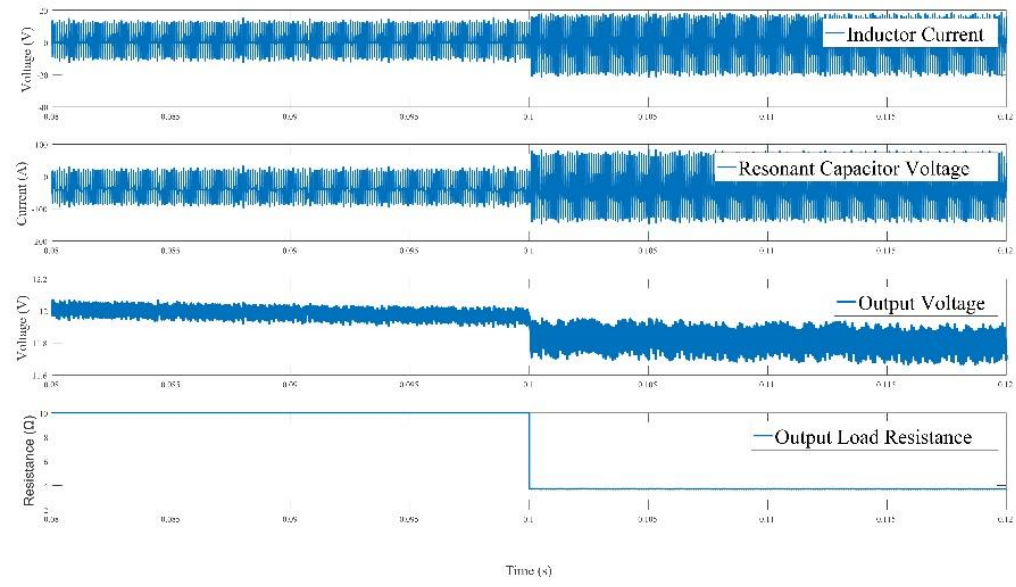

Figure 8: SRC controlled with PI controller. The operating point of the circuit was $48 \mathrm{~V}$ input voltage, $12 \mathrm{~V}$ output voltage at $10 \Omega$ output load

The second applied controller was a Sliding Mode Controller (SMC) with the first two states as the sliding plane. In Fig. 9 the load perturbation, while in Fig. 10 the reference perturbation is shown. The response time of the closed loop system can be measured with the time constant of the controlled system. In case of the $\mathrm{SMC}$ this time is $\mathrm{T}_{\mathrm{SMC}}=3,2426 \mathrm{~ms}$. 


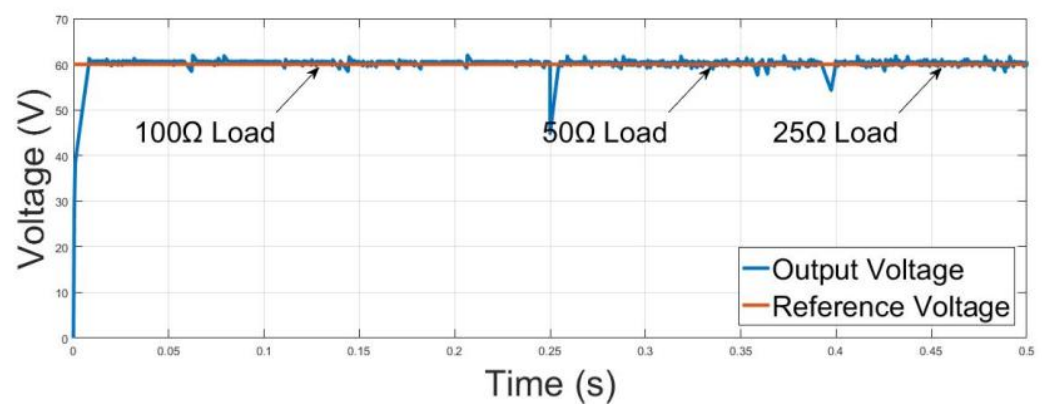

Figure 9: The load stability testing of the SRC

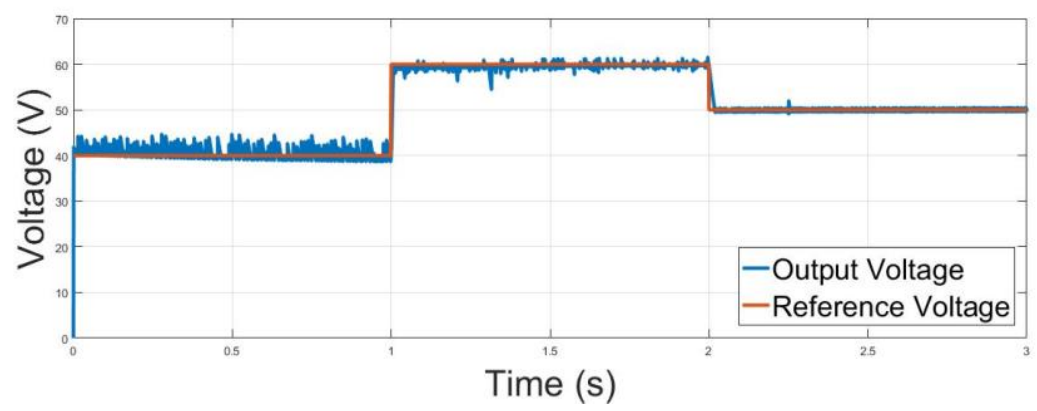

Figure 10: The reference tracking dynamics of the SRC

\section{Results and conclusions}

In this research we've studied the novel modelling technique named hybrid modelling, which incorporates continuous and discrete event design, to create a more precise representation of the described system. With the hybrid modelling technique, we successfully created a precise representation of the SRC circuit, which was tested and compared against the continuous model (Simscape/Power systems).

With the help of the hybrid model, we created controller structures of basic PI, SMC, and MPC, which were successfully tested both on the hybrid systems and the continuous models.

As final note we can state that the model predictive controller is ideal to track the reference voltage, and to create immunity to parameter perturbations, but not very adequate for fast transient response, and control without overshoot. If we want to achieve the same response time in case of the MPC as in case of the SMC the control horizon time should match the control time of the SMC, this time can be expressed in horizon samples $\left(f_{s} * T_{S M C}=1800\right)$ which would require an infeasible generation time and memory. 


\section{References}

[1] Lazar, M., Heemels, W. P. M. H., Weiland S., and Bemporad, A., "Stabilizing Model Predictive Control of Hybrid Systems," in IEEE Transactions on Automatic Control, vol. 51, no. 11, Nov. 2006, pp. 1813-1818.

[2] Witulski, A. F., Hernandez, A. F., and Erickson, R. W., "Small signal equivalent circuit modeling of resonant converters," in IEEE Transactions on Power Electronics, vol. 6, no. 1, Jan 1991, pp. 11-27.

[3] Sun, J., and Grotstollen, H., "Averaged modeling and analysis of resonant converters," Power Electronics Specialists Conference, 1993. PESC '93 Record, 24th Annual IEEE, Seattle, WA, 1993, pp. 707-713.

[4] Chung, H. S. H., Ionovici, A., and Zhang, J., "Describing functions of power electronics circuits using progressive analysis of circuit waveforms," in IEEE Transactions on Circuits and Systems I: Fundamental Theory and Applications, vol. 47, no. 7, Jul 2000, pp. 10261037.

[5] Borrelli, F., Bemporad, A., and Morari, M., "Predictive control for linear and hybrid systems", Cambridge University Press, 2017.

[6] Afshang, H., Tahami, F., and Molla-Ahmadian, H., "A novel hybrid modeling of DC-DC series resonant converters," IECON 2013 - 39th Annual Conference of the IEEE Industrial Electronics Society, Vienna, 2013, pp. 280-286.

[7] Salem, M., Jusoh, A., Idris, N. R. N., and Alhamrouni, I., "Modeling and simulation of generalized state space averaging for series resonant converter", in 2014 Australasian Universities Power Engineering Conference (AUPEC),, Sept 2014, pp 1-5. 